\section{Arsenic against the hedgehog}

\section{By Kai-Jye Lou, Staff Writer}

Researchers at the Stanford University School of Medicine have found that arsenic trioxide is able to antagonize the hedgehog pathway. ${ }^{1}$ The findings could open up hedgehog-driven solid tumors such as basal cell carcinoma and medulloblastoma as new indications for Cephalon Inc.'s Trisenox arsenic trioxide, which already is marketed for acute promyelocytic leukemia, a form of blood cancer.

Aberrant activation of the hedgehog $(\mathrm{Hh})$ pathway can result from loss-of-function mutations in patched 1 (PTCH1), which is an inhibitory cell surface receptor in the pathway, through activating mutations in smoothened (SMO), which is a membrane component downstream of PTCH1, or in intracellular pathway components downstream of SMO. ${ }^{2}$ Although a handful of biotechs are attacking SMO, a team led by Philip Beachy, a professor of developmental biology at Stanford and a member of the university's Institute for Stem Cell Biology and Regenerative Medicine, has been searching for ways to block downstream targets.

Beachy and colleagues decided to test arsenic trioxide (ATO) because arsenic-containing compounds are known to cause embryonic defects associated with deficiencies in Hh pathway signaling. The group found that ATO can antagonize Hh pathway signaling by decreasing the stability of glioma-associated oncogene homolog 2 zinc finger protein (GLI2) and preventing its accumulation in the primary cilium of the cell. GLI2 is a zinc finger transcription factor that acts downstream of SMO (see Figure 1, "A model for GLI2 trafficking and inhibition by ATO”).

In a mouse allograft model of Hh pathway-dependent medulloblastoma, research-grade ATO caused dose-dependent decreases in tumor growth compared with saline control. Importantly, a mutation that confers resistance to SMO antagonists did not significantly impair the ability of ATO to inhibit Hh pathway signaling.

Results were published in the Proceedings of the National Academy of Sciences.

"The key finding from our current study is that we have identified ATO as a hedgehog pathway antagonist," said Beachy, corresponding author on the paper. "The interesting property of ATO is that it doesn't act at the level of smoothened, which is what companies developing hedgehog pathway antagonists have been targeting. What we have shown in our current paper is that when you treat cells with arsenicals-and ATO in particular-certain aspects of hedgehog signaling that happen upstream of GLI2, like the accumulation of smoothened into the primary cilium, still occur normally."
The most advanced SMO inhibitors in the clinic include GDC-0449 from Roche's Genentech Inc. unit and partner Curis Inc., LDE225 from Novartis AG and IPI-926 from Infinity Pharmaceuticals Inc. and partner Mundipharma International Ltd. GDC-0449 is in Phase II testing for solid tumors, and LDE225 is in a Phase II trial for basal cell carcinoma (BCC). IPI-926 is in a Phase Ib/II trial for metastatic pancreatic cancer and in Phase I testing for advanced solid tumors. Chugai Pharmaceutical Co. Ltd., which is majority owned by Roche, licensed exclusive rights in Japan to GDC-0449 in February.

Other SMO inhibitors for solid tumors include BMS-833923 (XL139) from Bristol-Myers Squibb Co. and Exelixis Inc., which is in Phase I trials.

"The findings contribute to our understanding of the hedgehog pathway by describing an additional way downstream of smoothened to block hedgehog signaling," said Karen McGovern, director of cancer biology and head of the hedgehog biology group at Infinity. "The group performed technically challenging, well-controlled studies to conclusively show that arsenic-containing compounds block GLI2 mobilization to the cilia. They show that this block in signaling is specific and can lead to growth inhibition in the hedgehog pathway-dependent medulloblastoma model."

\section{Taking on resistance}

Targeting molecules downstream of SMO could help circumvent resistance to SMO antagonists, which can stem from genetic mutations in the target.

Thus, James Chen told SciBX that the PNAS article "is an exciting confirmation that targeting parts of the hedgehog pathway downstream of smoothened is feasible and has an anticancer effect in animal models."

In 2009, Chen and colleagues identified four small molecule $\mathrm{Hh}$ pathway signaling inhibitors that acted on GLI1, GLI2 or suppressor of fused homolog (SUFU), all of which are downstream of SMO in the Hh pathway. ${ }^{3}$ Chen is an associate professor in the Department of Chemical and Systems Biology at the Stanford University School of Medicine.

"Because arsenic trioxide is acting downstream of smoothened in the hedgehog pathway, the drug might be able to target a broader range of hedgehog-driven cancers, such as those with mutations that make them resistant to smoothened antagonists," said Wei Chen, an assistant professor in the Department of Medicine at the Duke University School of Medicine.

Infinity's McGovern noted, however, that the potency of ATO in the medulloblastoma mouse model "is not as dramatic as that seen with IPI-926 and smoothened inhibitors, which have been shown to cause frank regression of these tumors. This observation suggests that the potency of the arsenic compound will need to be improved upon before it could be used in the clinic to treat cancers driven by the hedgehog pathway."

Beachy thinks there is plenty of room in the armamentarium for multiple types of Hh pathway inhibitors and expects that ATO could be combined with a SMO antagonist to achieve stronger Hh pathway inhibition at lower drug concentrations. 


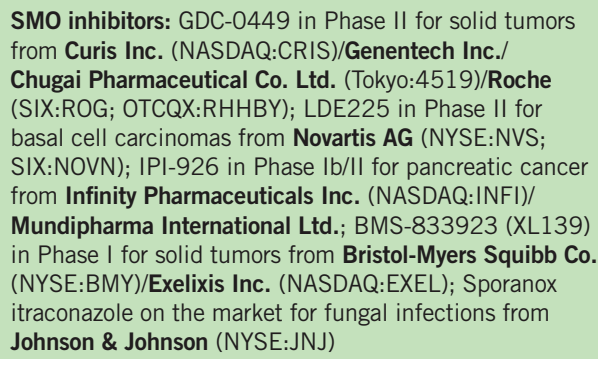

GLI2 inhibitors: Trisenox arsenic trioxide on the market for acute promyelocytic leukemia from Cephalon Inc. (NASDAQ:CEPH)

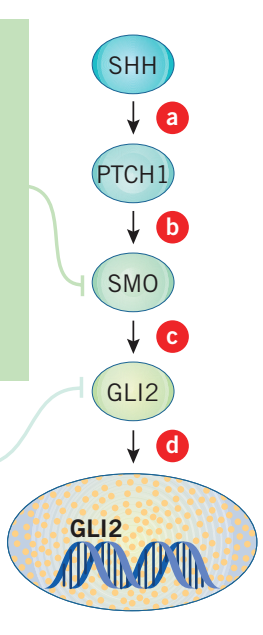

Figure 1. A model for GLI2 trafficking and inhibition by ATO. Stanford University School of Medicine researchers identified arsenic trioxide (ATO) and other arsenic-containing compounds as antagonists of the hedgehog pathway. They hypothesize that these compounds block the pathway by preventing the accumulation of glioma-associated oncogene homolog 2 zinc finger protein (GLI2) in the primary cilia.

The hedgehog $(\mathrm{Hh})$ pathway is activated when sonic hedgehog $(\mathrm{SHH})$ binds patched1 (PTCH1) [a], which in turn permits the release and activation of smoothened (SMO) [b]. Activated SMO accumulates in the primary cilium and alters the trafficking of GLI2, leading to the accumulation of the transcription factor GLI2 in this region $[c]$.

The altered trafficking results in the eventual transport of GLI2 into the nucleus in its active form [d]. Within the nucleus, activated GLI2 stimulates the transcription of tumor-promoting genes.

Earlier this year, the group showed that itraconazole, a commonly used systemic antifungal, antagonized Hh pathway signaling at the level of SMO and inhibited the growth of medulloblastoma tumors in mice. ${ }^{6}$

"One of the ideas that I think is really interesting is that it may be possible to combine arsenicals" with other compounds that modulate the pathway, Beachy told SciBX.

Indeed, in the PNAS paper, Beachy's group showed that ATO and the SMO antagonist cyclopamine had a synergistic effect.

"The nice thing about the synergistic activity between these compounds is that you could reduce the amount of each drug needed to achieve a therapeutic effect," said Stanford's Chen. He added that the use of lower doses also could reduce potential side effects and that combination therapy could decrease the likelihood of the cancer cells acquiring resistance.

The side effect issue is particularly important for ATO as the label for Trisenox warns of liver toxicities and cardiac QT prolongation. Because of the QT issue, it's been difficult to dose enough Trisenox to achieve efficacy in cancers other than APL. ${ }^{4}$

\section{GLI club}

McGovern and Duke's Chen think that an important next step would be to identify the exact target of ATO.

"There is still much more to be learned, as it is not fully understood what is the target of the arsenic compound and how it affects signaling through GLI2," McGovern told SciBX. "It would also be important to evaluate the activity of arsenic compounds in tumors that are not driven by mutations in the hedgehog pathway, especially in those where hedgehog signaling occurs between the tumor and its microenvironment."

Even in cancers that aren't driven by mutations in the Hh pathway, signaling in this pathway between the tumor and its microenvironment can drive processes that protect cancer cells from chemotherapeutics. ${ }^{5}$

Duke's Chen also wanted to see how ATO affects the translocation of GLI2 from the cytosol to the nucleus. "Because GLI2 is a transcription factor, it needs to enter the nucleus to carry out its function. Thus, studying the effects of this drug on GLI2 translocation from the cytosol to the nucleus could provide some additional insights on how the drug is affecting GLI2 function," he said.

Both McGovern and Stanford's Chen said they wanted to see studies in the mouse model to confirm that ATO and other arsenic-containing compounds can block the growth of cells that have become resistant to SMO antagonists. "It would also be important to test ATO in other types of hedgehog pathway-driven cancers like pancreatic and small cell lung cancers," Chen added.

Beachy's group is now evaluating the combination of arsenic-based compounds with other Hh pathway antagonists in mouse cancer models. He said future studies will seek to determine the serum and intratumor concentrations of ATO needed to achieve a therapeutic effect in $\mathrm{Hh}$ pathway-driven cancers.

Stanford has filed a patent application covering the use of arseniccontaining compounds in hedgehog-driven cancers. The work is available for licensing.

Cephalon declined to comment on the findings.

Lou, K.-J. SciBX 3(30); doi:10.1038/scibx.2010.911

Published online Aug. 5, 2010

\section{REFERENCES}

1. Kim, J. et al. Proc. Natl. Acad. Sci. USA; published online July 14, 2010; doi:10.1073/pnas.1006822107

Contact: Philip A. Beachy, Stanford University School of Medicine, Stanford, Calif. e-mail: pbeachy@stanford.edu

2. Epstein, E.H. Nat. Rev. Cancer 8, 743-754 (2008)

3. Hyman, J.M. et al. Proc. Natl. Acad. Sci. USA 106, 14132-14137 (2009)

4. Maggos, C. BioCentury 14(50), A10; Nov. 13, 2006

5. Bailey, J.M. et al. Clin. Cancer Res. 14, 5995-6004 (2008)

6. Kim, J. et al. Cancer Cell 17, 388-399 (2010)

COMPANIES AND INSTITUTIONS MENTIONED

Bristol-Myers Squibb Co. (NYSE:BMY), New York, N.Y. Cephalon Inc. (NASDAQ:CEPH), Frazer, Pa.

Chugai Pharmaceutical Co. Ltd. (Tokyo:4519), Tokyo, Japan

Curis Inc. (NASDAQ:CRIS), Cambridge, Mass.

Duke University School of Medicine, Durham, N.C.

Exelixis Inc. (NASDAQ:EXEL), South San Francisco, Calif.

Genentech Inc., South San Francisco, Calif.

Infinity Pharmaceuticals Inc. (NASDAQ:INFI), Cambridge, Mass.

Mundipharma International Ltd., Cambridge, U.K.

Novartis AG (NYSE:NVS; SIX:NOVN), Basel, Switzerland

Roche (SIX:ROG; OTCQX:RHHBY), Basel, Switzerland

Stanford University School of Medicine, Stanford, Calif. 